\title{
MODIFIED SPLIT HOPKINSON PRESSURE BAR FOR INVESTIGATIONS OF DYNAMIC BEHAVIOUR OF MAGNETORHEOLOGICAL MATERIALS
}

\author{
Leszek J. Frąś, Ryszard B. Pęcherski \\ Institute of Fundamental Technological Research of the Polish Academy of Science, Warsaw, Poland \\ e-mail:lfras@ippt.pan.pl
}

\begin{abstract}
The magnetorheological fluid is a functional material that is changing its rheological properties and finally solidifies in a magnetic field. The dynamic behaviour, tested with the use of the Split Hopkinson Pressure Bar is an important issue for description of this material, which is commonly used in different kinds of shock absorbers. This note presents a new idea how to modify the known SHPB set up in order to investigate dynamic properties of magnetorheological materials.
\end{abstract}

Keywords: Split Hopkinson Pressure Bar (SHPB), Magnetorheological Fluid (MRF), dynamic behaviour, solidification in magnetic field, ferroelements

\section{Introduction}

The magnetorheological fluid (MRF) is a material which is composed of microsized (less than $10 \mu \mathrm{m}$ diameter) magnetoactive carbonyl iron particles immersed in a carrier fluid, e.g. oil. The influence of the magnetic field changes properties of the MRF. The ferroelements are joined together and form characteristic shapes - braids, which are created along the magnetic field lines. The braids are concentrating in the process of sticking together. The applied magnetic field is changing the state of the material leading to solidification. Then the yield shear strength appears the important material parameter, the value of which is dependent on strength of the magnetic field. The aim of the paper is to present the idea how to investigate the behaviour of the solidified magnetorheological fluid under high strain rates. The experimental tests are carried out with application of the own construction of a laboratory test stand - the modified Split Hopkinson Pressure Bar (SHPB).

The Hopkinson Pressure bar was not only used to test metallic materials or brittle solids at high strain rates. Kenner (1980) adopted the split Hopkinson pressure bar to test pressure pulses in different kinds of fluids - ethyl alcohol, distilled water, glycerin and two kinds of oil. The experimental results help one to evaluate the one dimensional representation of pulse transmission and reflection at a solid-fluid interface. The results of experimental investigations make a basis for creation of a mathematical expression describing the pressure pulse distributed in the fluid. Another attempt to use the SHPB to test fluids was presented by Lim et al. (2009). The authors tested Cannon N4000 and N5100 fluids consisting 100\% polybutene. The experimental part was prepared with use of the SHPB furnished with aluminum bars and the fluid container was specially prepared. This part was machined of soft and flexible rubber and was tested with respect to fluid expansion in the radial direction. The dynamical tests of the polymer materials were considered by Siviour and Jordan (2016) where the behaviour of the rearrangement of microstructure of amorphous polymers was reviewed. The preliminary information about testing magnetorehological fluid under high strain rates was announced by Frąś (2015). There was presented a proposal of the laboratory set-up and an attempt to use the Perzyna viscoplasticity 
model to describe the behavior of magnetorheological fluids. Another attempt of testing magnetically controlled materials was presented by Wang (2016). The authors tested a self-prepared material with content of $75 \%$ carbonyl iron particles in several magnetic fields - form 95 to $382 \mathrm{kA} / \mathrm{m}$. During those experiments, stress values from 5 to almost $16 \mathrm{MPa}$ (for maximal magnetic field value) were obtained. Another attempt to use the Split Hopkinson Pressure Bar with magnetically controlled materials to test magnetorheological elastomers was presented by Liao et al. (2013). The authors used for several magnetic field strengths - maximally $320 \mathrm{kA} / \mathrm{m}$ and received in a dynamic compression test maximal stress values about $6 \mathrm{MPa}$ with velocity of the striker about $30 \mathrm{~m} / \mathrm{s}$. The microsized ferroelements in the magnetic field created the structure of viscoplastic solids. The structural rearrangements controlled by the magnetic field in the course of deformation can be investigated experimentally due to the recent developments of MEMS devices designed by Jarząbek et al. (2015).

\section{Magnetorheological fluid}

The magnetorheological fluid contains microsized ferroelements. The particles are spherically shaped and their diameter is less than $10 \mu \mathrm{m}$. The magnetoactive ferroelements are coated with a silicon shield and immersed in the carrying fluid, e.g. mineral oil. The silicon coating prevents from aggregation of particles which are made of a carbonyl iron material. The influence of the magnetic field forces the ferroelements to connect together into characteristic linear shapes the braids. The linearly-shaped elements made of ferroelements are sticking together and create the solid structure of the material.

(a)

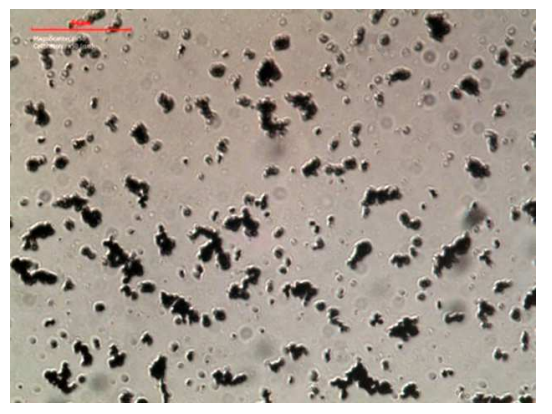

(b)

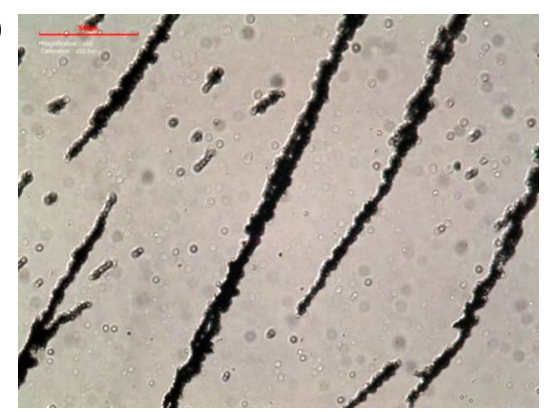

Fig. 1. The ferroelements in neutral state (a) and under the influence of a magnetic field (b)

The ferroelements can freely move in the carrying fluid. Figure $1 \mathrm{~b}$ shows the influence of the magnetic field on the way how the ferroelements are connected. The magnetoactive particles are sticking together creating characteristic braids parallel to the line of the magnetic field. The whole rearrangement of the structure during the deformation process can be described by tearing off a single ferroelement from the braid. The structure is deformed in the course of taking off from the sequence of separate braids.

The rearrangement of ferrorelements under the external force tends to swap the neighboring element. The particle is shifted and this produces a shear angle. The interaction energy of two particles is given as follows (Jolly et al., 1996)

$$
E_{12}=\frac{\left|m^{2}\right|\left(1-3 \cos ^{2} \theta\right)}{4 \pi \mu_{1} \mu_{0} r^{3}}
$$

where $m$ is mass of the particle, $\theta$ corresponds to the angle of shear, $r$ denotes the distance between particles and $\mu_{1}$ is the relative permeability, $\mu_{0}$ is vacuum permability. The shift of a 

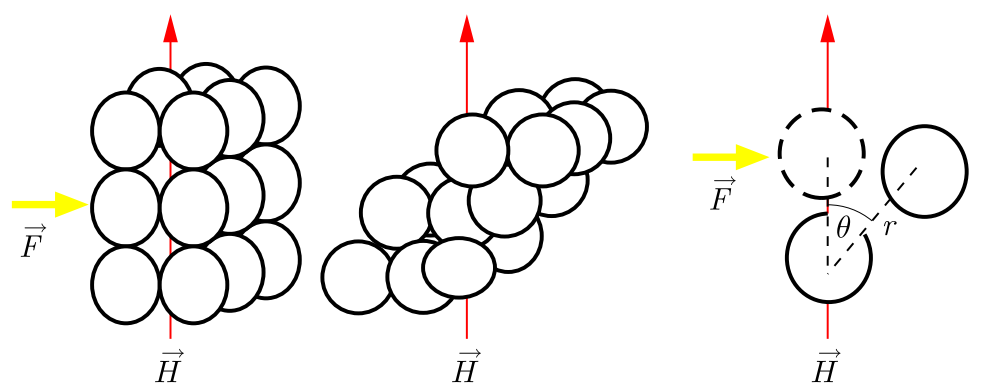

Fig. 2. The rearrangement of the structure, $F$ - external force, $H$ - magnetic flux

single ferroelement produces migration of the braid. The refreshable features of the magnetorheological fluid allow one to use them as an energy absorption material. The shifting of a single ferroelement by some external force causes reorganization of the skeleton structure.

\section{The laboratory test stand}

The testing stand is made on the basis of the Split Hopkison Pressure Bar with 7075 alloy aluminum bars. Their length is $1000 \mathrm{~mm}$ and diameter $20 \mathrm{~mm}$. The strain gauges are cemented in the middle - four on the each bar, and they are working as a quarter bridge sub system with the signal amplifier. The main idea is to modify the present laboratory device and prepare it to test the magnetorheological fluid - LORD MRF-140CG in a magnetic field at high strain rates by using the prepared coil - the resitivity of used copper $0.7 \mathrm{~mm}$ wire is $180 \mathrm{Ohm}$. It generates a $120 \mathrm{kA} / \mathrm{m}$ magnetic flux with a $360 \mathrm{~V}$ and $2 \mathrm{~A}$ direct current power supply. The coil with inner diameter $30 \mathrm{~mm}$ and length $50 \mathrm{~mm}$ has 80 turns of the wire. The power line is realized by EA-PS $8360-30$ and the maximum voltage is $360 \mathrm{~V}$ while the current is $50 \mathrm{~A}$. The laboratory device to investigate magnetorheological materials is subjected to modification. The idea of the proposed modification is presented in Fig. 3 .

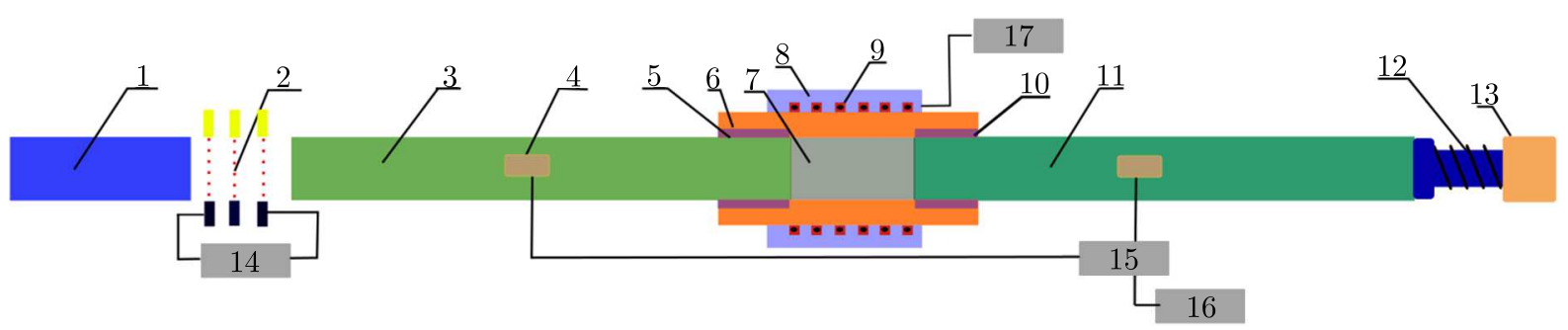

Fig. 3. 1 - striker, 2 - laser sensor to measure velocity, 3 - incident bar, 4 - strain gauges, 5 - glue, 6 - deformable hose, 7 - MR fluid, 8 - coil cover, 9 - coil , 10 - glue, 11 - transmitter bar, 12 - spring, 13 - momentum trap, 14 - velocity measure system, 15 - signal amplifier , 16 - oscilloscope, 17 - power supply for coils

The novelty of the proposed laboratory test stand is the application of coils for the sleeves shown in Fig. 3 to induce a constant magnetic field. Also a new shear testing device is designed. Due to this, the dynamic axial compression and shear tests of the solidified magnetorheological material is possible. The theory of measurement of the axial strain and determination of the axial stress with the use of the SHPB was presented by Klepaczko (2007). The striker accelerated by the gas gun achieves velocity $V_{0}$ which is measured by a matrix of diodes and photodiodes. In the case of impact by the striker against an incident bar shock wave is triggered. It has length $\varphi=2 L$, where $L$ is length of the striker. The time period of this wave is $T=2 L / C_{0}$, where $C_{0}$ is the velocity of the sound wave inside the bar. 


\section{Results}

During the experiment, the MR fluid solidified under the magnetic field responds to the stress waves. The effect of the compressive longitudinal incident pulse and the reflected pulse can be observed in Fig. 4b.

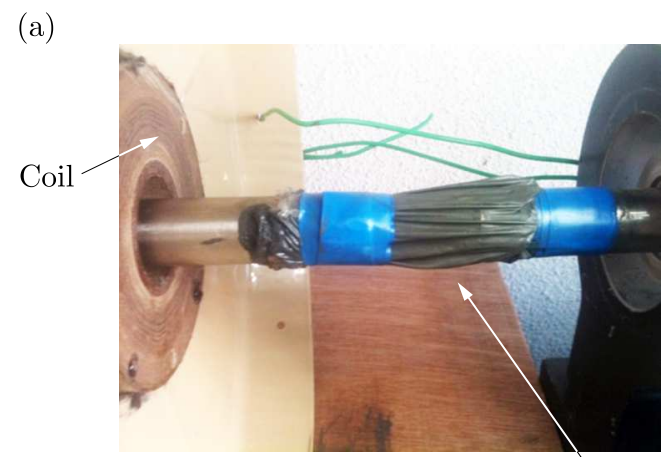

Specimen inside hose (b) Incident wave

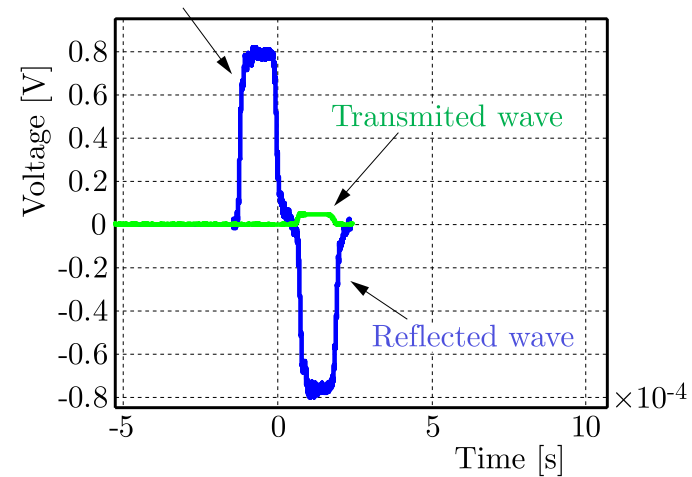

Fig. 4. (a) The deformable hose with the MR fluid before the test, (b) the observed wave forms of the tested material

The MR specimen is closed in a deformable hose made of latex with carefully prepared dimensions $-10 \mathrm{~mm}$ long and $20 \mathrm{~mm}$ in the external diameter of the bars, which allows one to keep about $3.2 \mathrm{ml}$ of the fluid injected with a syringe - Fig. 4a. It provides free deformation of the solidified MR fluid. The specially designed coil is generating a $120 \mathrm{kA} / \mathrm{m}$ magnetic flux and is responsible for sticking the ferroelements together. The generated field is parallel to the axis of incident - the transmitted bars what keeps the solidified ferroelements between the bars. The magnetoactive particles are arranged along the lines of the magnetic field. The deformable hose presented in Fig. 4 a with the MR fluid inside allows free deformation during the dynamical tests. The influence of the thin rubber hose on the longitudinal stress waves can be neglected. The results of experimental investigation are presented below.

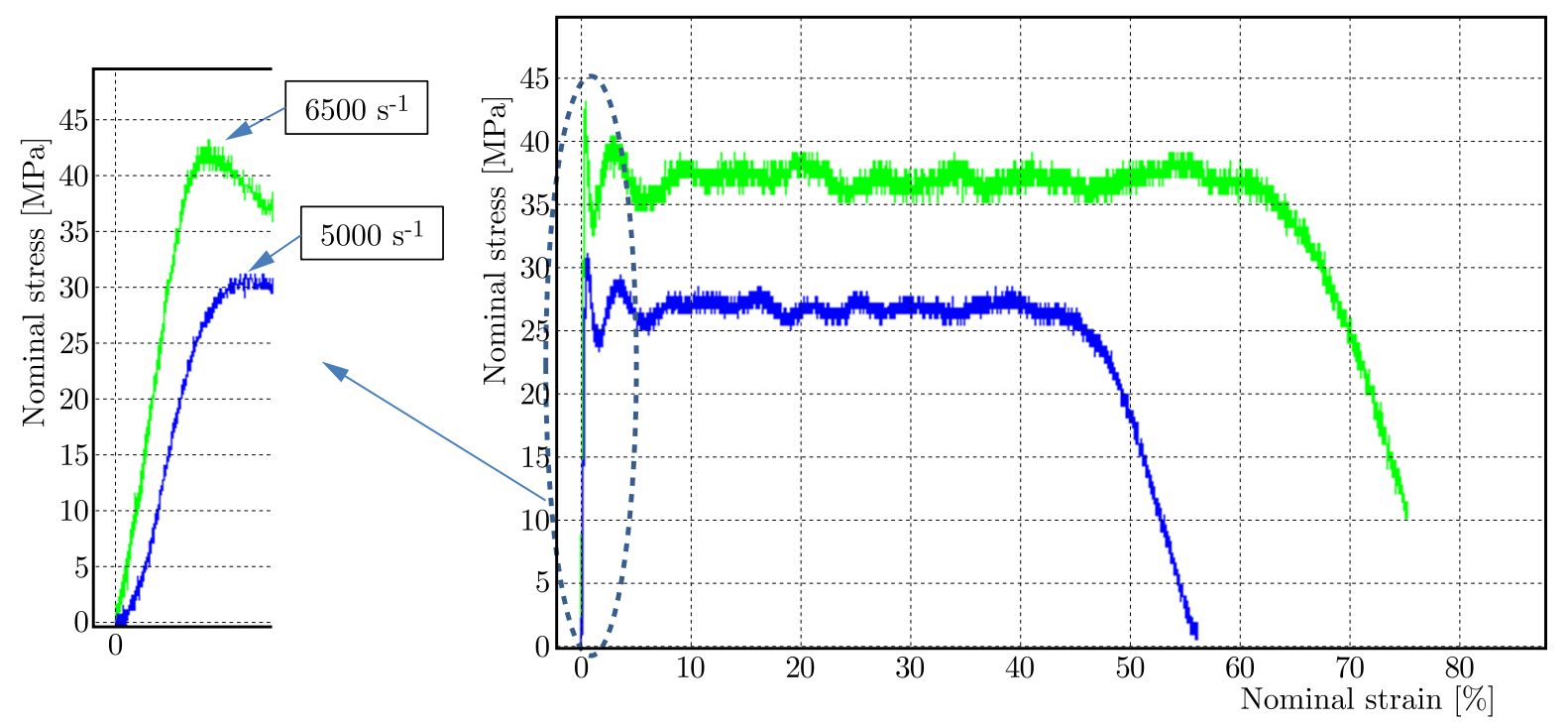

Fig. 5. The results of the experiment, the nominal stress-strain curve

The results presented in Fig. 5 are obtained during experimental investigation. The material has been tested with a $300 \mathrm{~mm}$ long striker and with two velocities $18 \mathrm{~m} / \mathrm{s}$ and $10 \mathrm{~m} / \mathrm{s} \pm 0.1 \mathrm{~m} / \mathrm{s}$. 
During the experiment aluminum bars without the shaper placed between the striker and the incident bar were used. The measured values were multiplied 200 times by the signal amplifier. For this reason, the obtained transmitted wave had an amplitude about $0.08 \mathrm{~V}$ with the velocity of the striker about $18 \mathrm{~m} / \mathrm{s}$ and about $0.035 \mathrm{~V}$ for $\sim 10 \mathrm{~m} / \mathrm{s}$.

\section{Conclusions}

The Split Hopkinson Pressure Bar has been adopted to test the magnetorheological fluid at high strain rates. The material was tested for two velocities of the striker to test and evaluate the laboratory set-up. The obtained results can be compared with the results of Wang et al. (2016) who tested similar materials but with a lower content of iron particles $74 \%$ versus $84 \%$ in our case. Wang et al. (2016) obtained for $200 \mathrm{mT}(159 \mathrm{kA} / \mathrm{m})$ and the strain rate $6000 \mathrm{~s}^{-1}$ the stress values in the range of 10-17 MPa. The material structure of Wang et al. (2016) was completely different ( $74 \%$ of carbonyl irons and several another additions closed into the gel structure) but the order of magnitude was similar to tested LORD MRF-140CG (84\% of carbonyl irons). The the effect of dispersion can play an essential role in the interpretation of the experimental data. The dispersion effect in the aluminium bars is not significant due to the large ratio of wavelength to the bar diameter, however, it has not been analysed yet and requires further studies. The comparison of our preliminary results confirms the validity of the concept of the proposed laboratory set-up and gives a promising perspective for a more detailed investigation aiming at the identification of Perzyna model.

Acknowledgment

This work was supported by the NCN (National Science Centre Poland) Research Project: 2015/17/N/ST8/02018.

\section{References}

1. FrĄŚ L.J., 2015, The Perzyna viscoplastic model in dynamic behaviour of magnetorheological fluid under high strain rates, Engineering Transactions (Rozprawy Inżynierskie), ISSN: 0867-888X, 63, 2, 233-243

2. Jarząbek D.M., Chmielewski M., Wojciechowski T., 2015, The measurement of the adhesion force between ceramic particles and metal matrix in ceramic reinforced-metal matrix composites, Composites Part A - Applied Science and Manufacturing, 76, 124-130

3. Jolly M.R., Carlson J.D., Munoz B.C., 1996, A model of the behaviour of magnetorheological materials, Smart Materials and Structures, 5, 607-614

4. Kenner V.H., 1980, The fluid Hopkinson bar, Experimental Mechanics, 20, 7, 226-232

5. Klepaczko J.R., 2007, Introduction to Experimental Techniques for Materials Testing at High Strain Rates, Institute of Aviation, Warsaw

6. Liao G., Gong X., Xuan S., 2913, Magnetic field-induced compressive property of magnetorheological elastomer under high strain rate, Industrial and Engineering Chemistry Research, 52, 25, 8445-8453

7. Lim A.S., Lopatnikov S.L., Gillespie JR. J.W., 2009, Phenomenological modelling of the response of a dense colloidal suspension under dynamic squeezing flow, Polymer Testing, 28, 891-900 
8. Siviour C.R., Jordan J.L., 2016, High strain rate mechanics of polymers: a review, Dynamic Behavior of Materials, 2, 15-32

9. Wang Y., Wang S., Xu C., Xuan S., Jiang W., Gong X., 2016, Dynamic behavior of magnetically responsive shear-stiffening gel under high strain rate, Composites Science and Technology, 127, $169-176$

Manuscript received August 24, 2017; accepted for print November 3, 2017 